\title{
A study of association between heart failure with preserved ejection fraction with hypertension and diabetes mellitus
}

\author{
Swapan Sarkar', Joydeep Biswas ${ }^{2}$, Suprotim Ghosh ${ }^{3}$ \\ ${ }^{1}$ Associate Professor and HOD, ${ }^{3}$ Postgraduate Resident, Department of General Medicine, Jagannath Gupta Institute \\ of Medical Sciences and Hospital, ${ }^{2}$ Associate Consultant, Department of Neurology, National Neurosciences Centre, \\ Kolkata, West Bengal, India
}

Background: Heart failure is a common clinical entity which we come across in our daily practice and accounts for significant mortality and morbidity. The basic pathophysiology lies in the inability of the heart to pump adequate blood (output) to meet the demands of circulation/tissue or can do so only at the expense of elevated left ventricular filling pressure. Among various types of heart failure, heart failure with preserved ejection fraction (HFpEF) is still a poorly understood entity and several comorbidities such as hypertension, diabetes, coronary artery disease, obesity, and CKD are common association of HFpEF. Diabetes causes heart failure by increasing the risk of CAD and by direct injury to myocardium (cardiomyopathy). Hence, in this cross-sectional observational study, we assess the cardiovascular risk factors such as hypertension and diabetes mellitus in association with HFpEF. Aims and Objectives: This study aims to establish the hypothesis that hypertension and diabetes mellitus are associated with a predictor of HFpEF. Materials and Methods: Ninety patients were selected. NTproBNP, HbA1C, FBS, PPBS level, and blood pressure was measured and echocardiogram was performed to assess ratio of transmitral flow velocity and annular velocity $\left(E / E^{\prime}\right)$; left ventricular end-diastolic pressure; and left ventricular ejection fraction (LVEF). Results: The mean age was $64 \pm 7$. Forty-two (46.67\%) were men and $48(53.33 \%)$ were female. Hypertension was present in $73(81.11 \%)$ and diabetes in $44(48.89 \%)$. E/E', a parameter of LV diastolic function, showed positive correlation to both risk factors in study $(r=0.653, p<0.001)$. Linear regression indicated that $E / E^{\prime}$ $(\beta$-coefficient $=0.845, p<0.001)$ was significantly associated with the presence of risk factors. Conclusion: The data show that the prevalence of HTN and DM is significantly higher in patients with HFpEF and establishes a strong association between duration of HTN and DM with symptomatic HFpEF.

Key words: Heart failure with preserved ejection fraction; Diastolic heart failure; NTproBNP; Ventricular ejection fraction; Diabetes complications; Hypertension.

\section{Access this article online}

Website:

http://nepjol.info/index.php/AJMS DOI: 10.3126/ajms.v12i12.40591

E-ISSN: 2091-0576

P-ISSN: 2467-9100

Copyright (c) 2021 Asian Journal of Medical Sciences

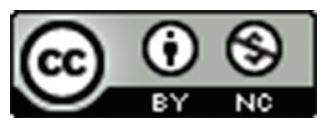

This work is licensed under a Creative Commons Attribution-NonCommercial 4.0 International License.

\section{INTRODUCTION}

Heart failure is a clinical syndrome that results from structural or functional impairment of ventricular filling or ejection of blood, which, in turn, leads to the cardinal clinical symptoms of dyspnea and fatigue and signs of heart failure, that is, edema and rales. ${ }^{1}$ Based on underlying mechanism, it could be divided into heart failure with preserved ejection fraction (left ventricular ejection fraction
$[\mathrm{LVEF}]>50 \%$, that is, heart failure with preserved ejection fraction $[\mathrm{HFpEF}]$ ) or heart failure with mid-range ejection fraction (LVEF 40-49\%, i.e., HFmrEF) or heart failure with reduced ejection fraction $(\mathrm{LVEF}<40 \%$, i.e., $\mathrm{HFrEF}){ }^{2}$ The number of cases of HFpEF has been increasing in the Western countries and consists more than $50 \%$ of total heart failure hospitalizations. The prevalence of $\mathrm{HFpEF}$ sharply increases with advancement of age, with a female predominance. ${ }^{3}$ There are limited data on heart failure in 
Indian population. Comparative data from Asian Heart Failure Registry showed that Indian patients with HFpEF were younger with mean age of 63.4 years in males and 46.4 years in females. Risk factors included hypertension $(40.3 \%)$ and DM $(28.8 \%)$. It has also been proved that HFpEF is prognostically as bad as HFrEF. ${ }^{4}$

\section{Aims and objectives}

To study the prevalence of HTN and DM in HFpEF.

\section{MATERIALS AND METHODS}

It was a prospective observational study among patients visiting outpatient department and IPD of Jagannath Gupta Institute of Medical Sciences and Hospital, a Medical College near Kolkata from 2020 to 2021 seeking advice in the Gen Medicine Dept. The study was pre-approved by Institutional Ethics Committee and the study was conducted after obtaining permission accordingly. Sample size was 90 patients between 30 and 90 years both male and female.

The risk factors in study, that is, hypertension and diabetes were based on the following criteria

1. Hypertension, that is, systolic blood pressure $>140 \mathrm{mmHg}$ and/or diastolic blood pressure $>90 \mathrm{mmHg}$ at least on two occasions or receiving antihypertensive drug

2. Diabetes, that is, history of type 2 diabetes diagnosed with American Diabetic Association criteria, that is, symptoms of diabetes plus random blood glucose concentration more than or equal to $200 \mathrm{mg} / \mathrm{dl}$ or, fasting plasma glucose more than or equal to $126 \mathrm{mg} /$ $\mathrm{dl}$ or, glycosylated hemoglobin more than or equal to $6.5 \%$ or, $2 \mathrm{~h}$ plasma glucose more than or equal to $200 \mathrm{mg} / \mathrm{dl}$ during an oral glucose tolerance test or were on medications for diabetes. ${ }^{5}$

\section{Inclusion criteria}

1. The diagnosis of HFpEF has been made based on the following criteria ${ }^{6,7}$

- Signs and symptoms of heart failure by clinical examination

- LVEF $>50 \%$ by echocardiography

- Echocardiographical evidence consistent with structural or functional anomaly including left diastolic dysfunction/increased left ventricular filling pressure or raised serum NTproBNP.

2. Age more than 30 years and $<90$ years.

\section{Exclusion criteria}

1. Presence of structural heart diseases, for example, hemodynamically significant valvular heart disease, prosthetic valve replacement, H/O HOCM, arrhythmias, implanted pacemaker, post-CABG, or peripheral vascular diseases
2. Pre-existing renal disease/CKD (eGFR $<60 \mathrm{ml} / \mathrm{min}$ )

3. History of cocaine or heroin use in the past 6 months

4. History of significant alcohol intake

5. Body mass index $(\mathrm{BMI})<18.5$ or $>40$

6. Severe anemia $(\mathrm{Hb}<8 \mathrm{~g} \%)$.

The patients who satisfied inclusion and exclusion criteria have been identified and included in this study. Proper history including demographic details, specific comorbidities, duration of HTN and DM, and medication details was taken and detailed clinically examination was done. For further clinical evaluation, a 12-lead ECG with long rhythm strip, straight X-ray skiagram of chest, and routine blood investigations such as complete blood count, renal function test, glycosylated hemoglobin, fasting plasma glucose, and ser. NTproBNP was performed.

Finally, transthoracic echocardiogram was performed with M-mode, 2D (two-dimensional), Doppler, and tissue Doppler imaging using standard techniques. At first, the following parameters were measured by M-mode: Interventricular septal thickness, left ventricular posterior wall thickness, end-systolic dimension of left atrium (LAD), and left ventricular internal diameter (LVID) at end diastole (LVIDd) and end systole (LVIDs). The LVEF was estimated by $2 \mathrm{D}$ approximation and wall motion abnormalities were noted, if any. Next, the following LV diastolic function parameters were measured by recording transmitral flow velocity using Doppler echocardiography, that is, peak early-diastolic transmitral flow velocity (E), peak latediastolic transmitral flow velocity (A), deceleration time, and E/A ratio. Then, tissue Doppler echocardiography was performed at medial mitral annulus. Peak early (E') and late $\left(A^{\prime}\right)$ diastolic mitral annular velocities and their ratio $\left(E^{\prime} / A^{\prime}\right)$ were measured. The ratio of transmitral flow velocity and annular velocity (E/E') was calculated to assess LV end-diastolic pressure (LVEDP) which was used as a parameter of LV diastolic dysfunction. Elevated filling pressure was based on $E / E$ ' ratio $>10$. Diastolic dysfunction was classified into four grades as per ASE guidelines $^{8}$ as tabulated (Table 1).

\section{Statistical analysis}

Quantitative data thus obtained have been analyzed and exported to statistical software SPSS V.27-2020 for proper interpretation. The continuous variables will be presented as mean \pm standard deviation, performed by Student's t-test or variance analysis. Percentage analysis was used to describe distribution of demographic variables. The association between HFpEF with diabetes and hypertension was obtained by Chi-square test separately and binary logistic regression analysis was done between predictor variable and dichotomous variable (hypertensive/diabetic or not). $\mathrm{P}<0.05$ was considered as statistically significant. 
Table 1: LV diastolic dysfunction as per LV relaxation, filling pressures, and 2D and Doppler findings ${ }^{9}$

\begin{tabular}{lcccc}
\hline Echo parameter & Normal & Grade I & Grade II & Grade III \\
\hline LV relaxation & Normal & Impaired & Impaired & Impaired \\
LAP & Normal & Low or normal & Elevated & Elevated \\
Mitral E/A ratio & $\geq 0.8$ & $\leq 0.8$ & $>0.8$ to & $>2$ \\
Average Ele' ratio & $<10$ & $<10$ & $10-14$ & $>14$ \\
Peak TR velocity $(\mathrm{m} / \mathrm{s})$ & $<2.8$ & $<2.8$ & $>2.8$ & $>2.8$ \\
LA volume index & Normal & Normal or increased & Increased & Increased \\
\hline
\end{tabular}

\section{RESULTS}

A total of 90 patients between 30 and 90 years both male and female who met the inclusion criteria were selected by simple random sampling. Majority belonged to the age group of $60-70$ years with mean age $64 \pm 7$ years. Forty-two (46.67\%) were men and $48(53.33 \%)$ were female. Hypertension was present in $73(81.11 \%)$ and diabetes in $44(48.89 \%)$ patients. Majority had ejection fraction between 55 and $60 \%$ and mean was $58.3 \pm 5.3 \%$; mostly with normal or near normal systolic function. The elevated mean LVMI indicated LV hypertrophy and decreased mean E/A indicated LV diastolic dysfunction, often produced by hypertension or diabetes. Mean E/E' was 9.78 \pm 4 .4. All variables were normally distributed by Kolmogorov-Smirnov goodness-of-fit test other than LVEF, E/A, E'/A', and E/E'.

Grade-2 (DD2) diastolic dysfunction patients were maximum in number (44, i.e., $48.89 \%$ ) followed by Grade-1 (DD1) diastolic dysfunction (38, i.e., $42.22 \%$ ), and only eight persons (i.e., 8.89\%) had Grade-3 (DD3) diastolic dysfunction (Figure 1). The prevalence of comorbidities in study (i.e., hypertension or diabetes) is progressively increasing along with the severity of diastolic dysfunction (from Grade-1 to Grade-2) to a fact that all patients having DD-3 were having at least one comorbidity and most of them have both (Figure 2). Apart from this, age of the patient and duration of hypertension/diabetes seemed to be an important determining factor.

\section{DISCUSSION}

In our study, high prevalence of hypertension (and also diabetes) in HFpEF was the most significant finding and signifies a strong etiological association (Figure 3). Patients having longer duration of HTN/DM or having both together were shown to have advanced DD with elevated LVEDP along with advancement of age. These findings are consistent with other large scale trials where hypertension has been identified as the commonest risk factor ${ }^{10}$ presenting in $50-90 \%$ of patients of $\mathrm{HFpEF}$, and prevalence is even more than that of $\mathrm{HFrEF}^{11,12}$ To study individual etiological association of HTN/DM with HFpEF, we also excluded certain other confounding risk factors such as chronic kidney disease, atrial fibrillation,

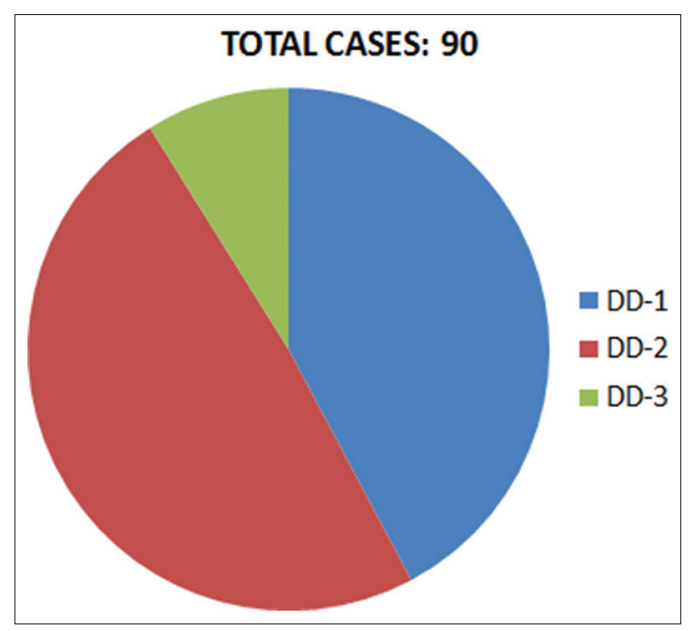

Figure 1: Severity of diastolic dysfunction

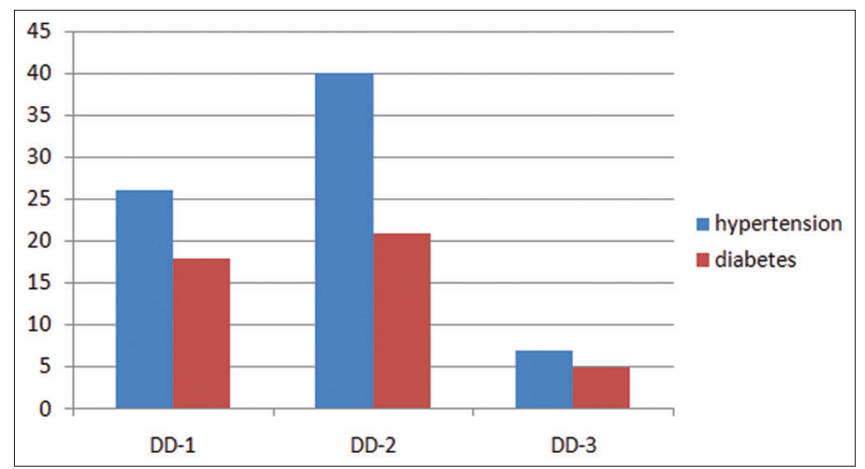

Figure 2: Comparison of number of cases

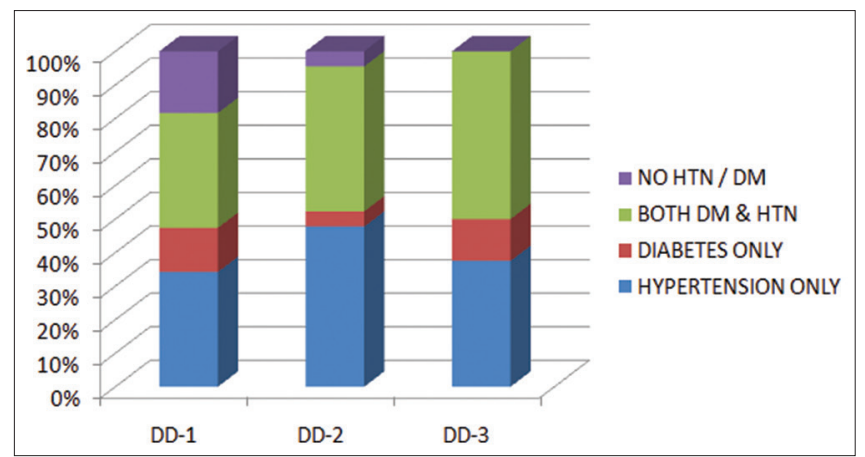

Figure 3: Relative prevalence of comorbidity with severity of diastolic dysfunction

and coronary artery disease which are a complication of HTN/DM itself and also being an important risk factor for HFpEF. ${ }^{13,14}$ 
Limitations of the study

$\mathrm{E} / \mathrm{E}^{\prime}$ was measured at septal mitral annulus. However, some studies recommended measuring velocities of lateral mitral annulus or averaged velocities of lateral and septal mitral annulus.

The inherent variability of risk factors such as alcohol intake, smoking, exercise, medications, and other unknown variables was not included in the study.

Finally, a multicenter trial with larger population is needed to further investigate etiological factors of $\mathrm{HFpEF}$ and its association with comorbidities.

\section{CONCLUSION}

Etiology and treatment approach of HFpEF differs from that of HFrEF. Moreover, HTN and DM are the modern day epidemics. Hence, if further studied by multicenter, prospective, longitudinal studies, this association may be used to identify the population at risk for $\mathrm{HFpEF}$ and to establish new targets for the management of diastolic dysfunction at the herald of its onset and prevention of symptomatic HFpEF resulting in longer survival and better prognosis.

\section{ACKNOWLEDGMENT}

To find out the association between risk factors (i.e. HTN and DM) with the severity of diastolic dysfunction as a predictor of $\mathrm{HFpEF}$.

\section{REFERENCES}

1. Jameson LA, Fauci AS, Kasper DL and Hauser SL. In: Longo DL and Loscalzo J, editors. Harrison's Principles of Internal Medicine. $20^{\text {th }}$ ed. New York: Mc Graw Hill; 2018.

2. Bozkurt B, Coats A, Tsutsui $H$ and Abdelhamid C. Universal definition and classification of Heart Failure. Eur J Heart Failure. 2021;23(6):352-380.

https://doi.org/10.1002/ejhf.2115

3. Oktay A, Rich $\mathrm{J}$ and Shah S. The emerging epidemic of heart failure with preserved ejection fraction. Curr Heart Fail Rep. 2013;7(10):401-410. https://doi.org/10.1007/s11897-013-0155-7

4. Lam C, Donal E and Vasan R. Epidemiology and clinical course of heart failure with preserved ejection fraction. Eur J Heart Fail. 2011;13(2):18-28.

https://doi.org/10.1093/eurjhf/hfq121

5. Jameson LA, Fauci AA and Kasper DL. In: Longo DL and Loscalzo J, editors. Harrison's Principlesof Internal Medicine. $20^{\text {th }}$ ed. New York: McGraw Hill; 2018.

6. Pieske B, Tschope $\mathrm{C}$ and de Boer R. How to diagnose heart failure with preserved ejection fraction. Eur Heart J. 2019;6(40):3297-317.

https://doi.org/10.1093/eurheartj/ehz641

7. Reddy $\mathrm{Y}$, Carter R and Obokata M. A simple evidence based approch to help guide diagnosis of heart failure with preserved ejection fraction. Circulation. 2018;20(138):861-870.

https://doi.org/10.1161/CIRCULATIONAHA.118.034646

8. Benzamin BF, Klein AL, Jae $K$ and Nagueh SF. Recommendations for the evaluation of left ventricular diastolic function by echocardiography: An update from the american society ofechocardiography and the european association of cardiovascular imaging. J Am Soc Echocardiogr. 2016;5(29):277-314

https://doi.org/10.1016/j.echo.2016.01.011

9. Naguesh M, Chair SA, Appleton M and Christopher P. Recommendations for the evaluation of left ventricular diastolic function by echocardiography. J Am Soc Echocardiogr. 2009;9(22):107-131.

https://doi.org/10.1080/08911762.2016.1245519

10. Dubourg O, Gueret $P$ and Beauchet A. Study of systolic and diastolic heart failure in french elderly population. Int $\mathrm{J}$ Cardiol. 2008;36(124):188-192.

https://doi.org/10.1016/j.ijcard.2007.01.050

11. Berry C, Doughty R, Granger $C$ and Kober L. The survival of patients with heart failure with preserved or reduced left ventricular ejection fraction: An individual patient data metaanalysis. Eur Heart J. 2012;33(14):1750-1757. https://doi.org/10.1093/eurheartj/ehr254

12. Massie B, Carson $P$ and McMurray J. Irbesartan. N Engl J Med. 2008;28(359):2456-2467.

https://doi.org/10.1056/NEJMoa0805450

13. Brouwer FP, de Boer RA, van der Harst $P$ and Voors $A A$. Incidence and epidemiology of new onset heart failure with preserved vs reduced ejection fraction in a community based cohort: 11 year follow up of PREVEND. Eur Heart J. 2013;6(34):1424-1431. https://doi.org/10.1093/eurheartj/eht066

14. Maisel W and Stevenson L. Atrial fibrillation in heart failure: Epidemiology, pathophysiology and rationale for therapy. Am J Cardiol. 2003;32(91):2D-8D. https://doi.org/10.1016/S0002-9149(02)03373-8

\footnotetext{
Authors Contribution:

SS- Concept and design of study; and guidance for preparation of manuscript; JB- Review of literature, preparation of draft, and interpretation of result; SG- Collection of data and interpretation of results, preparation of $1^{\text {st }}$ draft.

Work attributed to:

Jagannath Gupta Institute of Medical Sciences and Hospital, Kolkata - 700 137, West Bengal, India.

Orcid ID:

Dr. Swapan Sarkar - (10 https://orcid.org/0000-0003-0007-052X

Dr. Joydeep Biswas - https://orcid.org/0000-0002-2312-284X

Dr. Suprotim Ghosh - (i) https://orcid.org/0000-0002-2632-0980

Source of Funding: None, Conflicts of Interest: None.
} 